\title{
A Low-Field Mobility Model for Bulk, Ultrathin Body SOI and Double-Gate n-MOSFETs With Different Surface and Channel Orientations-Part I: Fundamental Principles
}

\author{
Luca Silvestri, Susanna Reggiani, Member, IEEE, Elena Gnani, Member, IEEE, \\ Antonio Gnudi, Member, IEEE, and Giorgio Baccarani, Fellow, IEEE
}

\begin{abstract}
An easy-to-implement electron mobility model that accurately predicts low-field mobility in the channel of bulk MOSFETs and UTB-SOI FETs fabricated on different crystal orientations is developed. The model accounts for the influence of surface orientation and in-plane current-flow direction on effective masses, subband repopulation, and scattering rates. The paper is divided into two parts. In Part I, the general features of the model are presented, taking into account phonon, Coulomb, and surface roughness scattering. Band and repopulation effects are addressed based on the solution of the Schrödinger-Poisson equations. The effects of interface states and ultrathin body are treated in Part II.
\end{abstract}

Index Terms-Crystal orientation, mobility model, SOI MOSFETs, ultrathin silicon.

\section{INTRODUCTION}

$\mathbf{T}$ HE AGGRESSIVE downscaling of CMOS devices is reaching intrinsic limitations and needs new technological solutions. Ultrathin body (UTB) devices with either planar or vertical architectures such as single-gate silicon-on-insulator (SG-SOI) FETs, double-gate (DG) FETs, FinFETs, and silicon nanowires $(\mathrm{SNW})$ are the most promising candidates for fabricating sub-50-nm devices [1]. The study of the device performance requires the development of predictive physical models for carrier transport. For this purpose, both mobility models [2] and enhanced drift-diffusion models, which account for quasi-ballistic transport, have been proposed [3]. To further improve the device performance for future technology nodes, attention is being given to the carrier mobility, which has been proven to play an important role on device performance even for ultrashort gate lengths [4], [5].

Several techniques to optimize carrier mobility in UTB FETs such as strain [6] and suitable surface orientations [7] are still under investigation. Furthermore, structures such as rectangular

Manuscript received October 28, 2009; revised April 2, 2010; accepted April 13, 2010. Date of publication May 20, 2010; date of current version June 23, 2010. This work was supported by the EU Noe 216171 (NANOSIL) via the IU.NET Consortium. The review of this paper was arranged by Editor C. McAndrew.

The authors are with the Department of Electronics (DEIS) and E. De Castro Advanced Research Center for Electronic Systems (ARCES), University of Bologna, 40136 Bologna, Italy (e-mail: 1silvestri@ arces.unibo.it).

Color versions of one or more of the figures in this paper are available online at http://ieeexplore.ieee.org.

Digital Object Identifier 10.1109/TED.2010.2049210
SNW, FinFETs, or Tri-gate FETs usually exhibit sidewall transport on the (110) crystallographic planes [8]-[11]. Thus, a deep comprehension of the physical details related with the different crystallographic orientations is required.

In order to scale UTB FETs down to the ultimate technology nodes, the silicon body needs to be thinned below $5 \mathrm{~nm}$ to suppress short-channel effects, thus raising a concern on device variability and transport limitations. It has been experimentally demonstrated that electron mobility is a sensitive function of the silicon-body thickness, especially when $t_{\mathrm{Si}}$ is below $5 \mathrm{~nm}$ [12]-[17].

The carrier mobility of single-gate (SG) and double-gate (DG) UTB MOSFETs has been extensively investigated, and a TCAD model has been proposed [2]. However, a TCAD model for UTB MOSFETs with unconventional surface and currentflow orientations is missing, and to the author's knowledge, only experimental investigations can be found.

The aim of this work is to derive a physically based mobility model for device simulation tools that accurately predicts the low-field electron mobility in SG and DG FETs with different surface and channel orientations and silicon thicknesses as small as $2.5 \mathrm{~nm}$. The mobility model presented in [2] has been modified to account for different crystallographic orientations.

The paper is divided into two parts. In Part I, a general description of the model is provided and its validation against experiments is illustrated. Starting from a mobility formulation for bulk MOSFETs as a function of the effective transverse field, doping density, and surface and channel orientations [18], a number of improvements have been added to reproduce the experiments taken on (100), (110)/ $/ 100\rangle$ and $(110) /\langle 110\rangle$ SG and DG FETs, with a silicon body thicker than $10 \mathrm{~nm}$. In Part II, important corrections for ultrathin body (thinner than $10 \mathrm{~nm}$ ) are considered.

The paper is organized as follows. In Section II, the generalized mobility model is discussed. The effective inversion-layer thickness model for unconventional surface orientations and ultrathin body is described in Section III, along with the phonon scattering model. Coulomb and surface-roughness scattering models for unconventional surface orientations are treated in Section IV. An accurate analytical description of the energy subbands, which includes nonparabolicity effects, is reported in Section V. Finally, conclusions are drawn in Section VI. 


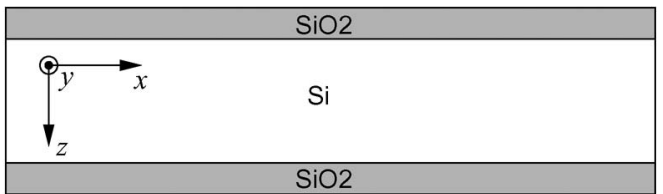

(a)
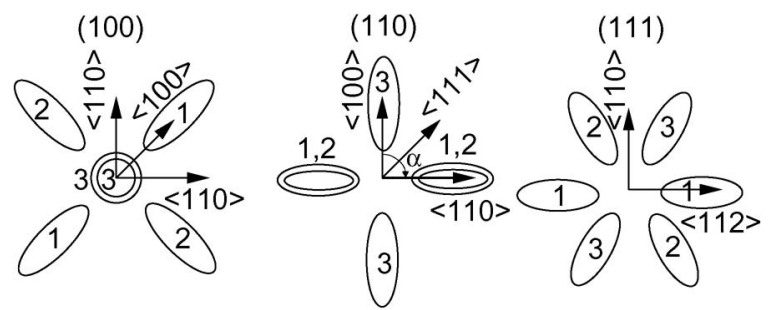

(b)

Fig. 1. (a) Orientation of the reference axes. (b) In-plane minima projections for (100), (110), and (111) surface orientations. The equivalent minima are labeled with the same number. The most relevant in-plane current flow directions are also indicated.

\section{Generalized Mobility Model}

Consider a silicon film on a substrate with one of the three crystallographic orientations shown in Fig. 1(b). The $z$-axis is set parallel to the structural confinement direction while carrier transport occurs along the $x$-axis, as shown in Fig. 1(a). The inversion-layer quantization, due to the combined effect of structural confinement and application of a transverse electric field, causes the formation of energy ladders. More specifically, (100)-oriented FETs exhibit two energy ladders: the lower one, referred to as "unprimed," is two-fold degenerate and originates from the valleys labeled " 3 ," while the upper one, referred to as "primed," is four-fold degenerate and is related with valleys " 1 " and "2." In (110) substrates, the "unprimed" ladder is related to valleys " 1 " and " 2 " while the primed one is related to the " 3 " valleys. Finally, a single six-fold degenerate ladder is formed along the (111) orientation.

In order to calculate the effective masses along the quantization $\left(m_{z}\right)$, the transport $\left(m_{x}\right)$, and the width $\left(m_{y}\right)$ directions for each valley in an arbitrary-oriented device, the generalized effective mass approach by [11] is followed. Starting from the ellipsoidal coordinate system (ECS), it is possible to determine the effective mass tensor in the device coordinate system (DCS) through the appropriate rotation of the ECS, followed by a rediagonalization process. Two different channel directions are analyzed for (110)-oriented samples, namely the $\langle 100\rangle$ and $\langle 110\rangle$ (see Fig. 1). The effective masses corresponding to the analyzed cases are reported in Table I.

The quantization leads to unequal relative populations of the different valley pairs. Therefore, in-plane transport is generally described by a 2-D tensorial effective mobility, which retains the anisotropy of the single-valley effective mobilities

$$
\hat{\mu}_{\mathrm{eff}}=\sum_{v=1}^{3} p_{v} \hat{\mu}_{v}
$$

where $\hat{\mu}_{v}$ and $p_{v}$ are the mobility tensor and relative population of the $v$ th valley, respectively. A similar formulation is already available in 3-D drift-diffusion transport simulation tools, which handle bulk piezoresistivity and in general, material anysotropy (see, e.g., [19] and [20]). Such tools can also be directly used in this case since the out-of-plane (normal to the interface) mobility component plays no role and provided that the in-plane effective mobility model (1) is implemented. Unfortunately, this approach is unpopular in commercial TCAD tools because the effective-mobility dependence on integral (non-local) electron concentration and electric field may lead to numerical problems. As an alternative, a local mobility tensor $\hat{\mu}$ that depends on the local normal electric field $E_{\perp}(z)$ and carrier concentration $n(z)$ can be defined, satisfying the following equation:

$$
\hat{\mu}_{\mathrm{eff}}=\frac{\int_{o}^{t_{\mathrm{Si}}}\left(n(z)-n_{0}(z)\right) \hat{\mu}\left(n, E_{\perp}\right) d z}{\int_{o}^{t_{\mathrm{Si}}}\left(n(z)-n_{0}(z)\right) d z}
$$

where $n(z)-n_{0}(z)$ is the excess electron concentration in the inversion layer. It should be noted that experiments measure only the $x x$ component of the mobility tensor.

We follow a two-step procedure in the model development. In the first step, we define an analytical model for the effective mobility (1) as a function of the effective electric field $E_{\text {eff }}$ and the inversion-charge concentration per unit area, calibrating the parameters on the experiments. $E_{\text {eff }}$ is calculated as

$$
E_{\mathrm{eff}}=\frac{\int_{0}^{t_{\mathrm{Si}}}\left(n(z)-n_{0}(z)\right) E_{\perp}(z) d z}{\int_{o}^{t_{\mathrm{Si}}}\left(n(z)-n_{0}(z)\right) d z} .
$$

In the second step, a local mobility model, which depends on $E_{\perp}(z)$ and $n(z)$, is provided as described in Section $\mathrm{V}$ of Part II.

The relative populations are calculated by assuming Boltzmann statistics as

$$
p_{v}=\frac{m_{d_{v}} \exp \left(-E_{C_{v}} / k_{B} T\right)}{\sum_{v=1}^{3} m_{d_{v}} \exp \left(-E_{C_{v}} / k_{B} T\right)}
$$

where $m_{d_{v}}=\sqrt{m_{x_{v}} m_{y_{v}}}$ is the density-of-states effective mass of the 2DEG in the $v$ th valley, $E_{C_{v}}$ is the valley bottom energy, $k_{B}$ is the Boltzmann constant, and $T$ is the lattice temperature. In Section V, it will be shown that the subband bottom energies are calculated analytically, accurately reproducing the solution of the Schrödinger-Poisson problem in the cross section normal to the transport direction. Following [21], the single-valley mobility tensor is modeled as

$$
\hat{\mu}_{v}=\mu_{v} \hat{m}_{v}^{-1}, \quad \hat{m}_{v}^{-1}=\left(\begin{array}{cc}
m_{0} / m_{x_{v}} & 0 \\
0 & m_{0} / m_{y_{v}}
\end{array}\right)
$$

where $m_{0}$ is the free-electron mass and $\hat{m}_{v}^{-1}$ is the inverse scaled mass tensor of a 2DEG, defined for each valley $v$ to account for the anisotropy effects induced by different in-plane crystal directions. This is especially needed when considering 
TABLE I

PRincipal EFFECTIVE MASSES FOR A 2DEG IN (100)-, (110)-, AND (111)-ORIENTED SAMPLES $\left(m_{l}=0.916 m_{0}\right.$ AND $\left.m_{t}=0.19 m_{0}\right)$

\begin{tabular}{|c|c|c|c|c|}
\hline (Wafer) $/<$ channel $\rangle$ & $m_{x}$ & $m_{y}$ & $m_{z}$ & valleys (see fig. 1) \\
\hline$(100) /\langle 100\rangle$ & $m_{l}$ & $m_{t}$ & $m_{t}$ & $m_{t}$ \\
& $m_{t}$ & $m_{l}$ & $m_{l}$ \\
& $m_{t}$ & $\left(m_{l}+m_{t}\right) / 2$ & $2 m_{l} m_{t} /\left(m_{l}+m_{t}\right)$ \\
& $m_{t}$ & $m_{t}$ & $m_{t}$ \\
\hline$(110) /\langle 100\rangle$ & $m_{l}$ & $m_{t}$ & $2 m_{l} m_{t} /\left(m_{l}+m_{t}\right)$ & $m_{t}$ \\
\hline$(110) /\langle 110\rangle$ & $\left(m_{l}+m_{t}\right) / 2$ & $m_{l}$ & 3 \\
\hline$(111) /\langle 112\rangle$ & $(2 / 3) m_{t}\left[\left(2 m_{l}+m_{t}\right) /\left(m_{l}+m_{t}\right)\right]$ & $\left(m_{l}+m_{t}\right) / 2$ & $3 m_{l} m_{t} /\left(2 m_{l}+m_{t}\right)$ \\
& $\left(2 m_{l}+m_{t}\right) / 3$ & $m_{t}$ & $3 m_{l} m_{t} /\left(2 m_{l}+m_{t}\right)$ \\
\hline
\end{tabular}

the unprimed 4-fold valleys in (110) samples. Finally, $\mu_{v}$ is calculated accounting for the different scattering mechanisms combined via Matthiessen's rule

$$
\mu_{v}=\frac{q}{m_{0} \sum_{j} \tau_{v_{j}}^{-1}} .
$$

In (6), $q$ is the elementary charge and $\tau_{v_{j}}$ is the average momentum relaxation time (MRT) due to the $j$ th scattering mechanism for the $v$ th valley, as discussed in the next sections.

\section{PHONON SCATTERING}

Following the approach of [2], the phonon-limited inverse MRT relative to the $v$ th valley is defined as

$$
\frac{1}{\tau_{\mathrm{PS}, v}}=\frac{C_{v} m_{d_{v}}}{W_{v}}
$$

where $W_{v}$ is the effective width of the electron distribution in the $v$ th valley and $C_{v}$ is a constant related to the intravalley acoustic-phonon scattering parameters. Intervalley scattering is not considered in view of the low-field regime, which is close to equilibrium. By considering that the largest fraction of the electron population of each subband occupies the subband's bottom, we define the average effective width as

$$
W_{v}=\frac{\sum_{i} p_{i, v}\left(\sum_{j \leq i} F_{i, j}^{v}\right)^{-1}}{\sum_{i} p_{i, v}}
$$

where indexes $i, j$ identify the subbands of the $v$ th valley; $p_{i, v}$ is the $v$ th subband population, and $F_{i, j}^{v}$ is the form factor relative to the $(i, j)$ subband couple, which is defined as

$$
F_{i, j}^{v}=\int_{0}^{t_{\mathrm{Si}}}\left|\Psi_{i, v}(z)\right|^{2}\left|\Psi_{j, v}(z)\right|^{2} d z
$$

where $\Psi_{i, v}(z)$ are the electron eigenfunctions.

The numerical computation of the effective widths for (110) and (111) surface orientations has been carried out by means of our Schrödinger-Poisson solver [22] generalized to singlegate and double-gate SOI MOSFETs with different crystal orientations. The eigenfunctions are zero at the $\mathrm{Si} / \mathrm{SiO}_{2}$ interfaces. Neumann boundary conditions are applied to the bottom edge of the buried oxide in SG-SOI FETs. The calculated $W_{v}$ are shown in Figs. 2 and 3 versus the effective field $E_{\text {eff }}$ for

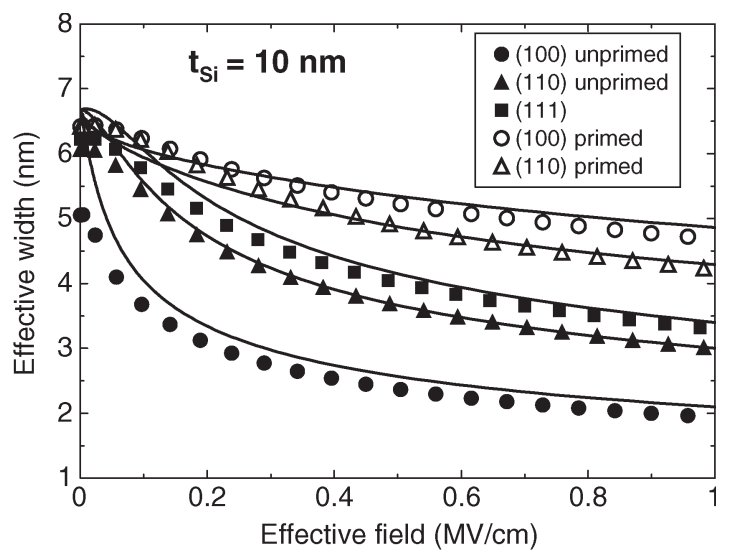

Fig. 2. Effective widths for the unprimed and primed subband ladders as a function of the effective field at $t_{\mathrm{Si}}=10 \mathrm{~nm}$. Symbols: numerical computations. Solid lines: (10).

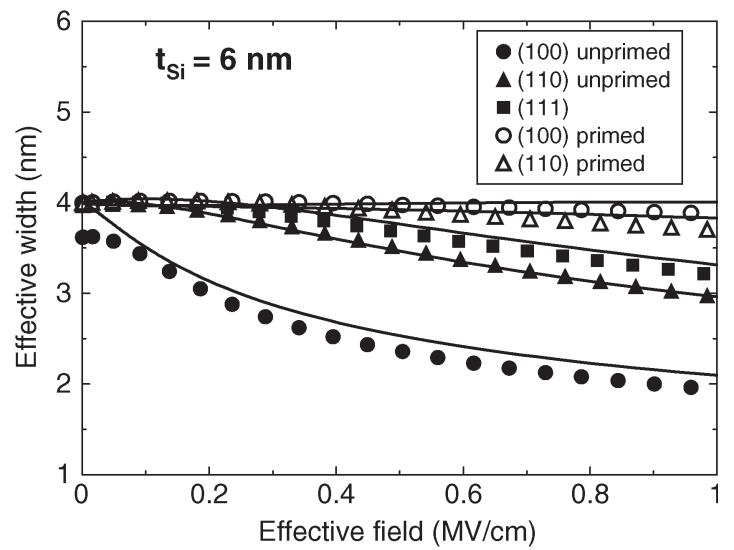

Fig. 3. Effective widths for the unprimed and primed subband ladders as a function of the effective field at $t_{\mathrm{Si}}=6 \mathrm{~nm}$. Symbols: numerical computations. Solid lines: (10).

different surface orientations in SG-SOI FETs with $t_{\mathrm{Si}}=10$ and $6 \mathrm{~nm}$, respectively. According to [2], the average effective width is modeled as

$$
W_{v}=\frac{W_{\mathrm{T} v}}{\left[1+\left(W_{\mathrm{T} v} / W_{\mathrm{E} v}\right)^{4}\right]^{1 / 4}}
$$

where

$$
\begin{aligned}
& W_{\mathrm{T} v}=\frac{2}{3} t_{\mathrm{Si}}+W_{T 0 v}\left(\frac{t_{\mathrm{Si}}}{t_{\mathrm{Si} 0}}\right)^{4}\left(\frac{E_{\mathrm{eff}}}{E_{\mathrm{eff} 0}}\right) \\
& W_{\mathrm{E} v}=W_{E 0 v}\left(\frac{E_{\mathrm{eff}}}{E_{\mathrm{eff} 0}}\right)^{-\gamma} .
\end{aligned}
$$


$W_{\mathrm{T} v}$ represents the effective width of the confined electron gas at low effective fields and small silicon thicknesses; $W_{\mathrm{E} v}$ is the effective width at large normal fields and thick SOI films. In (11) and (12), $t_{\mathrm{Si} 0}=10^{-7} \mathrm{~cm}, E_{\text {effo }}=10^{6} \mathrm{~V} / \mathrm{cm}$, $W_{T 0 v}$, and $W_{E 0 v}$ are fitting parameters calibrated on numerical results (see the solid lines of Figs. 2 and 3 ). By comparing the average widths of the primed and unprimed subband ladders, a slighter dependence on the electric field is observed due to the higher energy levels, which are less sensitive to it. $\gamma$, whose theoretically predicted value is $1 / 3$ [23], is found to be equal to 0.29 for the (111) orientation and for the unprimed ladders of (100) and (110) while the values of 0.17 and 0.2 have been found for the primed ladders of (100) and (110), respectively. As shown in Figs. 2 and 3, the difference between the unprimed and primed effective widths is more pronounced in (100) than in (110) samples. This effect can be due to the smaller difference between the quantization masses of the unprimed and primed valleys in the (110) case, leading to closer primed and unprimed energy levels.

\section{Coulomb And Surface Roughness Scattering}

The Coulomb scattering term is modeled as

$$
\frac{1}{\tau_{\mathrm{CS}, v}}=C_{\mathrm{CS}_{0}}\left(\frac{N_{\mathrm{inv0}}}{N_{\mathrm{inv}}}\right)\left(\frac{N_{A}}{N_{A 0}}\right)^{\sigma}
$$

where $N_{A}$ is the substrate doping density and $N_{A 0}=$ $10^{17} \mathrm{~cm}^{-3}, N_{\text {inv0 }}=10^{13} \mathrm{~cm}^{-2}, C_{\mathrm{CS}_{0}}$, and $\sigma$ are the fitting parameters, which are extracted by comparing the analytical model with experiments for bulk MOSFETs [23] with different $N_{A}$ [18]. The inversion-layer electron density $N_{\text {inv }}$ for the bulk MOSFETs with uniform $N_{A}$ measured in [23] can be calculated by inverting the usual expression

$$
E_{\mathrm{eff}}=\left(q / \epsilon_{\mathrm{Si}}\right)\left(\eta N_{\mathrm{inv}}+N_{\mathrm{depl}}\right)
$$

with $N_{\text {depl }}$ as the depletion charge per unit area, $\epsilon_{\mathrm{Si}}$ as the silicon permittivity, and $\eta$ as a constant equal to $1 / 2$ for (100) and $1 / 3$ for (110) and (111) substrates, as shown in [23]. In (14). $N_{\text {depl }}$ is calculated as

$$
N_{\mathrm{depl}}=\sqrt{4 \epsilon_{\mathrm{Si}} \Phi_{B} N_{A} / q}
$$

with $\Phi_{B}=k_{B} T \log \left(N_{A} / N_{i}\right)$ as the Fermi potential and $N_{i}=$ $8.765 \times 10^{9} \mathrm{~cm}^{-3}$ as the intrinsic carrier concentration at $300 \mathrm{~K}$. It is worth noting that when implementing the mobility model in a TCAD tool like, e.g., the 1-D quantum driftdiffusion solver for SOI MOSFETs described in [24], $E_{\text {eff }}$ and $N_{\text {inv }}$ are directly obtained from the numerical results.

The empirical formulation of surface roughness scattering under the assumption of single subband occupation reads

$$
\frac{1}{\tau_{\mathrm{SR}, v}}=C_{\mathrm{SR}_{0}} m_{d_{v}}\left(\frac{E_{\mathrm{eff}}}{E_{\mathrm{eff} 0}}\right)^{\delta}
$$

where $C_{\mathrm{SR} 0}$ is a constant and $\delta=2$ [23]. We use here the same expression, with $C_{\mathrm{SR} 0}$ as a fitting parameter and $\delta=2.7$ for (100), 1.5 for (110), and 1 for (111) orientations as found from the experiments. The different effects on mobility of Coulomb and surface roughness scattering for different orientations was predicted by Monte Carlo simulations [25], and was ascribed to the different energy quantization and interface properties. This result is not surprising since the density of surface atoms and avaiable bonds strongly depend on the crystal orientation [26], [27].

\section{BAND StRuctures AND REPOPUlation EFFECTS}

In order to compute the relative populations of the unprimed and primed ladders in (100)- and (110)-oriented samples and their dependence on the silicon film thickness and the effective field, we develop an analytical formulation based on physical considerations. In (111) samples, a single six-fold degenerate ladder is present; hence, no repopulation occurs. For zero normal electric field (quantum well), the analytical solution of the Schrödinger equation provides the expression for the energy levels. The relative distance between the primed and unprimed subband edges reads

$$
\Delta E_{\mathrm{CT}}=E_{\mathrm{CT}}^{\prime}-E_{\mathrm{CT}}=\frac{(\hbar \pi)^{2}}{2 t_{\mathrm{Si}}^{2}}\left(\frac{1}{m_{z}^{\mathrm{pr}}}-\frac{1}{m_{z}^{\mathrm{unpr}}}\right) .
$$

From (17), the separation between the energy minima of the two ladders increases with the reduction of $t_{\mathrm{Si}}$, and electrons mostly populate the unprimed ladder. At large electric fields, on the other hand, the energy minima can be theoretically calculated assuming a triangular potential well [28] as

$$
\begin{aligned}
E_{\mathrm{CE}_{v}} & =\left(\sqrt[3]{\frac{9}{32}}+\sqrt[3]{\frac{9}{4}}\right) \sqrt[3]{\frac{\hbar^{2} q^{2}}{m_{z_{v}}}} E_{\mathrm{eff}}^{2 / 3} \\
& =E_{\mathrm{CE} 0_{v}} \sqrt[3]{\frac{m_{0}}{m_{z_{v}}}}\left(\frac{E_{\mathrm{eff}}}{E_{\mathrm{eff} 0}}\right)^{\zeta}
\end{aligned}
$$

We adopt the last expression and use $E_{\mathrm{CEO}_{v}}$ and $\zeta$ as fitting parameters to accuratly reproduce the energy minima of bulk MOSFETs on (100) and (110) substrates, as computed by the Schrödinger-Poisson solver. Indicating with $\Delta E_{\mathrm{CE}}$, the difference between the ladder minima at high electric fields, the behavior of $\Delta E_{C}$ can be expressed as

$$
\Delta E_{C}=\Delta E_{\mathrm{CT}}\left[1+\left(\Delta E_{\mathrm{CE}} / \Delta E_{\mathrm{CT}}\right)^{\beta}\right]^{1 / \beta}
$$

where $\beta=3.5$. Figs. 4 and 5 compare (19) with numerical simulation results provided by our Schrödinger-Poisson solver for different silicon thicknesses, effective fields, and substrate orientations. The above model for the energy difference between the subband edges of the primed and unprimed ladders allows us to compute the relative valley populations from (4), which are shown in Fig. 6 versus $t_{\mathrm{Si}}$ for (100)-oriented samples. According to previous works [17], [29], a clear repopulation effect occurs at about $t_{\mathrm{Si}}=7 \mathrm{~nm}$. The unprimed ladder, which exhibits the lower transport effective mass (see Table I), turns out to be fully populated for $t_{\mathrm{Si}} \leq 4 \mathrm{~nm}$, with a beneficial effect on mobility. 


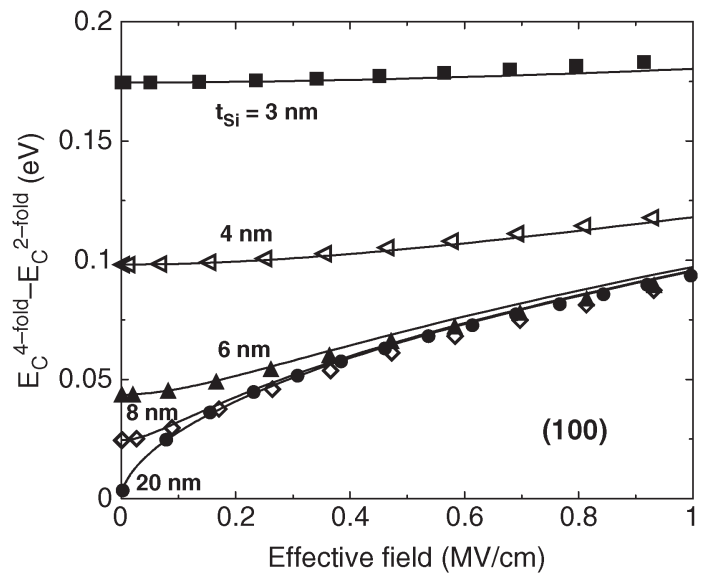

Fig. 4. Difference between the conduction subband edges of the 2-fold and 4-fold valleys in (100) samples versus the effective normal field for SG-SOI FETs with different silicon thicknesses. Symbols: numerical values; solid lines: (19).

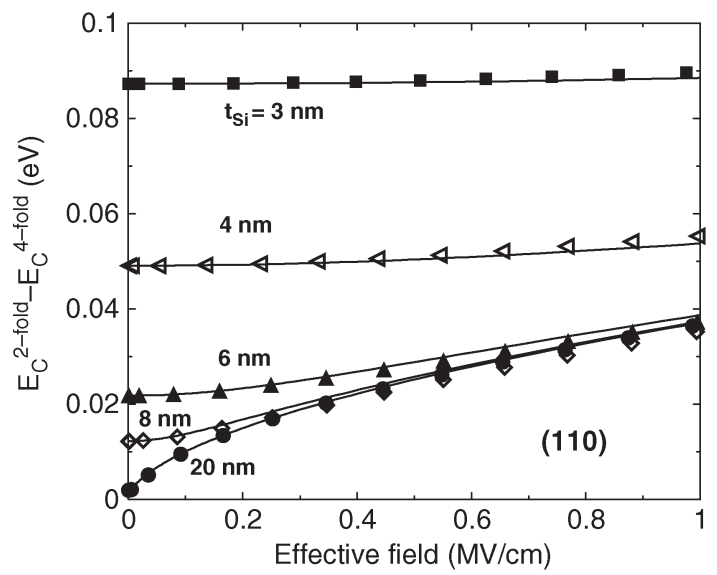

Fig. 5. Difference between the conduction subband edges of the 2-fold and 4 -fold valleys in (110) samples versus the effective normal field for SG-SOI FETs with different silicon thicknesses. Symbols: numerical values; solid lines: (19).

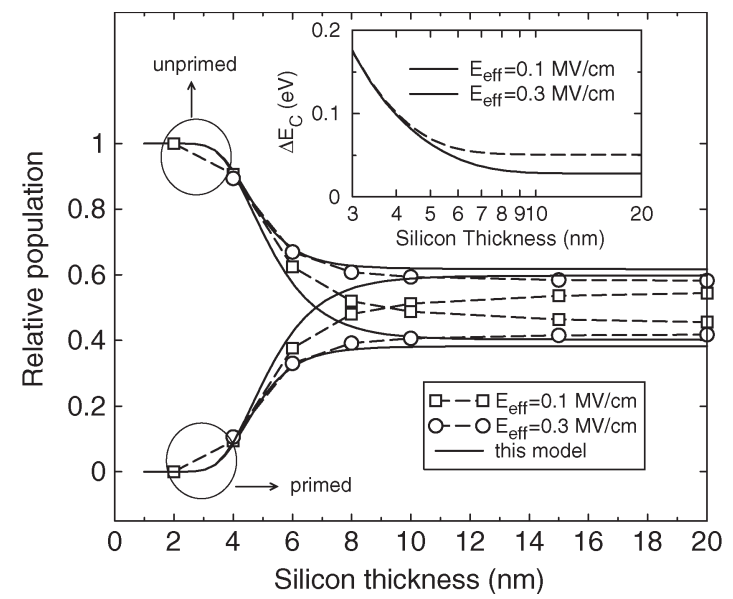

Fig. 6. Relative occupancy of the unprimed and primed subband ladders in (100)-oriented SG-SOI samples versus the silicon thickness for two different effective fields from (4). Symbols: numerically calculated relative populations; lines: this model. Inset: energy shift between primed and unprimed ladders from (19).

\section{A. Anisotropy and Nonparabolicity in the (110) Orientation}

Further band-structure analyses are required for (110) substrates. As already pointed out, the effective mobility in (110) samples exhibits a strong in-plane anisotropy. Consider, for example, a $\langle 100\rangle$-oriented FET channel on a (110) substrate. As shown in Fig. 1(b), the unprimed ladder is the most populated one and it exhibits a higher mobility due to the low transport effective mass (see Table I). On the contrary, if the FET channel is $\langle 110\rangle$-oriented, the transport effective mass of the four-fold ladder is higher. This clarifies the experimental mobility reduction with respect to that of the $\langle 100\rangle$-oriented FET channel [30].

An additional effect has been outlined in the experimental analysis carried out by Uchida in [30]. The strong nonparabolicity of the conduction band in the [110] crystalline direction, i.e., the quantization direction for the (110) orientation, leads to a smaller subband-edge difference than predicted by (19) between the unprimed and primed ladders for energies exceeding $100 \mathrm{meV}$. In order to account for this effect, (17) and (19) have been modified. We assume that nonparabolicity effects play a relevant role in the 2-fold primed ladder, mainly because of the high energy minimum. At zero field, the lowest energy of the primed ladder in the parabolic-band approximation is

$$
E_{\mathrm{CT}}^{\prime}=\frac{(\hbar \pi)^{2}}{2 t_{\mathrm{Si}}^{2} m_{z}^{\mathrm{pr}}}
$$

According to [31], a nonparabolic energy dispersion relationship for the conduction band minimum can be expressed as

$$
E_{\mathrm{CT}}^{\prime \mathrm{NP}}\left(1+\alpha E_{\mathrm{CT}}^{\prime \mathrm{NP}}\right)=\frac{\left(\hbar k_{z}\right)^{2}}{2 m_{z}^{\mathrm{pr}}}
$$

where $\alpha$ is the nonparabolicity factor and $k_{z}$ is the wave vector component in the quantization direction. When $k_{z}=\pi / t_{\mathrm{Si}}$ and using (20), (21) yealds

$$
E_{\mathrm{CT}}^{\prime \mathrm{NP}}=\frac{-1+\sqrt{1+4 \alpha E_{\mathrm{CT}}^{\prime}}}{2 \alpha} .
$$

The $\alpha$ value has been fitted on Uchida's nonparabolic energy dispersion relationship [30] shown in the inset of Fig. 7, and has been fixed at $3.4 \mathrm{eV}^{-1}$.

A second correction is made in order to account for nonparabolicity effects at high electric fields. From (18) the energy minima have an increasing trend with $E_{\text {eff }}$ due to the parabolic-band approximation. In order to avoid an overestimation of $\Delta E_{C}$, we modify (19) as follows:

$$
\Delta E_{C}^{\mathrm{NP}}=\Delta E_{C}\left[1-\log \left(1+\frac{N_{\mathrm{inv}}}{9 \times 10^{12}}\right)\right]
$$

and reproduce the difference of the subband edges of the primed and unprimed ladders calculated in [30], as shown in Fig. 7. At low $N_{\text {inv }} \Delta E_{C}^{\mathrm{NP}}$ increases by decreasing $t_{\mathrm{Si}}$ up to about $4 \mathrm{~nm}$, decreases for $t_{\mathrm{Si}}$ between 4 and $2.5 \mathrm{~nm}$, where it becomes zero and unprimed and primed ladders cross each other (see Fig. 7). The effects of anisotropy and nonparabolicity on experimental mobilities for $(110) /\langle 100\rangle$ and $(110) /\langle 110\rangle$ devices are shown in Fig. 8. The enhancement of the 4-fold 


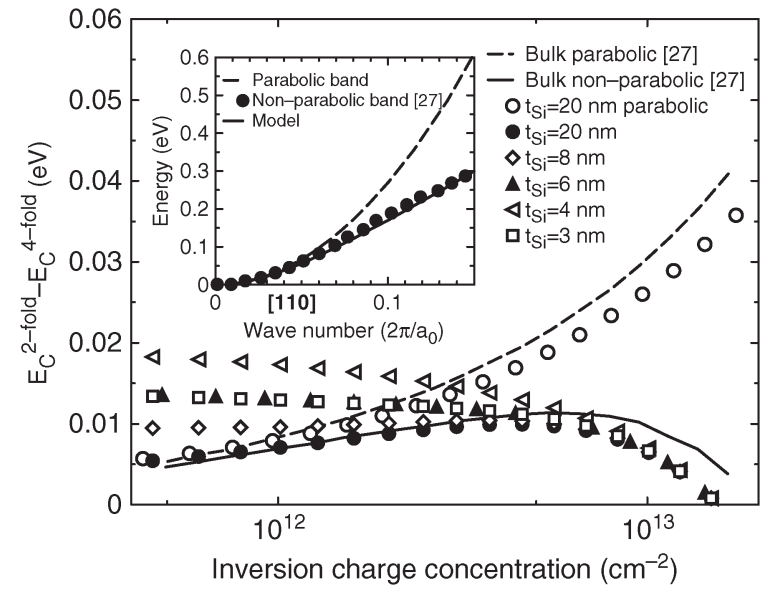

Fig. 7. Difference of conduction-subband edges between the 2-fold and 4-fold valleys of (110)-oriented substrates versus the inversion charge concentration for SG-SOI FETs with different silicon thicknesses after nonparabolic corrections (22) and (23). The parabolic and nonparabolic bands of a bulk MOSFET calculated in [30] are also shown. Inset: $E(k)$ relationship of energy ellipsoids "3" in Fig. 1 along [110] crystal orientation calculated in [30] (symbols) and from (22) (solid line).

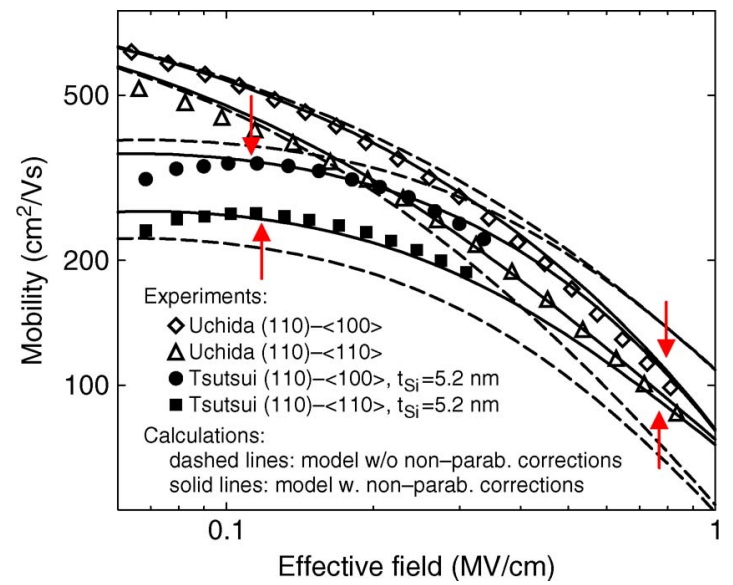

Fig. 8. Electron mobility in bulk and SG-SOI MOSFETs on (110) substrates versus the effective field for $\langle 100\rangle$ and $\langle 110\rangle$ channel directions. Symbols: experiments from [16] and [30]. Solid lines: this model with nonparabolic corrections. Dashed lines: this model with parabolic bands. Arrows highlight the different impact on mobility of the two nonparabolic corrections in (22) and (23) for small $t_{\mathrm{Si}}$ and high electric fields, respectively.

ladder population, which is favorable for the $\langle 100\rangle$ and unfavorable for the $\langle 110\rangle$ channel directions, has a strong impact on mobility if a parabolic band model is used (dashed lines). The nonparabolic corrections to $\Delta E_{C}$ (solid lines) make the subband repopulation less effective.

As shown in Fig. 8, the application of the nonparabolic corrections to $\Delta E_{C}$ is required to reproduce the experimental results. It is worth noting that the effective width in (8) is still based on a parabolic-band approximation, but in the lowmedium $E_{\text {eff }}$ range and for $t_{\mathrm{Si}}>5 \mathrm{~nm}$, the nonparabolicity effects on carrier mobility are negligible.

The physical effects considered so far allow us to reproduce the experimental mobilities in [13] and [16] with $t_{\mathrm{Si}}$ as small as about $5 \mathrm{~nm}$, as shown in Fig. 9. However, to further improve the fitting of the (110) experiments, the scattering induced by interface states in SG-SOI FETs has to be included, as explained in Part II. Unfortunately, no experimental investigations are

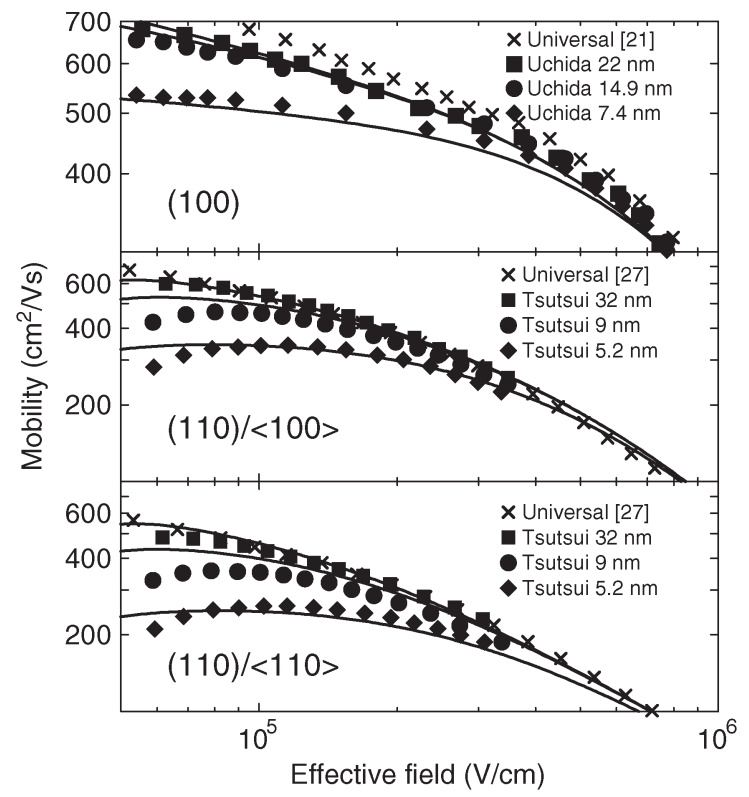

Fig. 9. Electron mobility in (100), (110)/ $\langle 100\rangle$, and $(110) /\langle 110\rangle$ SG-SOI FETs versus effective field for various silicon thicknesses. Symbols: experiments in [13] and [16]. $\mu$ versus $N_{\text {inv }}$ curves in [16] have been converted in $\mu$ versus $E_{\text {eff }}$ curves assuming $N_{A}=5 \times 10^{15} \mathrm{~cm}^{-3}$.

available in the literature to validate the mobility model in the (111) SOI case.

\section{CONCLUSION}

A low-field electron mobility model suitable for devicesimulation tools in (100) and (110) UTB-SOI MOSFETs has been developed and calibrated on a wide set of experimental data. The model accounts for the main physical effects related to the quantum-mechanical structural confinement such as valley repopulation, in-plane anisotropy, and nonparabolicity, and transposes them into simple analytical formulations. The correct description of the band structure (energy levels and effective masses) is shown to be fundamental to predict mobility in UTB devices for any arbitrary in-plane current-flow direction.

\section{REFERENCES}

[1] International Technology Roadmap for Semiconductors 2007 Edition. [Online]. Available: http://public.itrs.net

[2] S. Reggiani, E. Gnani, A. Gnudi, M. Rudan, and G. Baccarani, "Lowfield electron mobility model for ultra-thin-body SOI and double-gate MOSFETs with extremely small silicon thicknesses," IEEE Trans. Electron Devices, vol. 54, no. 9, pp. 2204-2212, Sep. 2007.

[3] C. Kampen, A. Burenkov, J. Lorenz, H. Ryssel, V. Aubry-Fortuna, and A. Bournel, "An application-driven improvement of the drift-diffusion model for carrier transport in decanano-scaled CMOS devices," IEEE Trans. Electron Devices, vol. 55, no. 11, pp. 3227-3235, Nov. 2008.

[4] R. Ohba and T. Mizuno, "Nonstationary electron/hole transport in sub$0.1 \mu \mathrm{m}$ MOS devices: Correlation with mobility and low-power CMOS application," IEEE Trans. Electron Devices, vol. 48, no. 2, pp. 338-343, Feb. 2001.

[5] M. Lundstrom, "On the mobility versus drain current relation for a nanoscale MOSFET," IEEE Electron Device Lett., vol. 22, no. 6, pp. 293-295, Jun. 2001.

[6] S. E. Thompson, S. Suthram, Y. Sun, G. Sun, S. Parthasarathy, M. Chu, and T. Nishida, "Future of strained Si/semiconductors in nanoscale MOSFETs," in IEDM Tech. Dig., 2006, pp. 1-4. 
[7] M. Yang, V. W. C. Chan, K. K. Chan, L. Shi, D. M. Fried, J. H. Stathis, A. I. Chou, E. Gusev, J. A. Ott, L. E. Burns, M. V. Fischetti, and M. Ieong, "Hybrid-orientation technology (HOT): Opportunities and challenges," IEEE Trans. Electron Devices, vol. 53, no. 5, pp. 965-978, May 2006.

[8] S. Mehrotra, A. Paul, M. Luisier, and G. Klimeck, "Surface and orientation dependence on performance of trigated silicon nanowire pMOSFETs," in Proc. IEEE Workshop Microelectron. Electron Devices, 2009, pp. 1-4.

[9] B. Yu, L. Chang, S. Ahmed, H. Wang, S. Bell, C. Yang, C. Tabery, C. Ho, Q. Xiang, T. King, J. Bokor, C. Hu, M. Lin, and D. Kyser, "FINFET scaling to $10 \mathrm{~nm}$ gate length," in IEDM Tech. Dig., 2002, pp. 251-254.

[10] J. Kavalieros, B. Doyle, S. Datta, G. Dewey, M. Doczy, B. Jin, D. Lionberger, M. Metz, W. Rachmady, M. Radosavljevic, U. Shah, N. Zelick, and R. Chau, "Tri-gate transistor architecture with high-k gate dielectrics, metal gates and strain engineering," in Proc. Symp. VLSI Technol. Dig. Tech. Papers, 2006, pp. 50-51.

[11] A. Rahman, M. Lundstrom, and A. Ghosh, "Generalized effective-mass approach for n-type metal-oxide-semiconductor field-effect transistors on arbitrarily oriented wafers," J. Appl. Phys., vol. 97, no. 5, p. 053702, Mar. 2005.

[12] K. Uchida, H. Watanabe, A. Kinoshita, J. Koga, T. Numata, and S. Takagi, "Experimental study on carrier transport mechanisms in ultrathin-body SOI n- and p-MOSFETs with SOI thicknesses less than $5 \mathrm{~nm}$," in IEDM Tech. Dig., 2002, pp. 47-50.

[13] K. Uchida, J. Koga, and S. Takagi, "Experimental study on carrier transport mechanisms in double- and single-gate ultrathin-body MOSFETs-Coulomb scattering, volume inversion, and $\delta \mathrm{T}_{\mathrm{SOI}}$-induced scattering," in IEDM Tech. Dig., 2003, pp. 805-808.

[14] D. Esseni, M. Mastrapasqua, G. K. Celler, C. Fiegna, L. Selmi, and E. Sangiorgi, "An experimental study of mobility enhancement in ultrathin SOI transistors operated in double-gate mode," IEEE Trans. Electron Devices, vol. 50, no. 3, pp. 802-808, Mar. 2003.

[15] G. Tsutsui, M. Saitoh, T. Saraya, T. Nagumo, and T. Hiramoto, "Mobility enhancement due to volume inversion in (110)-oriented ultra-thin body double-gate nMOSFETs with body thickness less than $5 \mathrm{~nm}$," in IEDM Tech. Dig., 2005, pp. 729-732.

[16] G. Tsutsui and T. Hiramoto, "Mobility and threshold-voltage comparison between (110)- and (110)-oriented ultrathin-body silicon MOSFETs," IEEE Trans. Electron Devices, vol. 53, no. 10, pp. 25822588, Oct. 2006

[17] D. Esseni, A. Abramo, L. Selmi, and E. Sangiorgi, "Physically based modeling of low field electron mobility in ultrathin single- and doublegate SOI n-MOSFETs," IEEE Trans. Electron Devices, vol. 50, no. 12, pp. 2445-2455, Dec. 2003.

[18] L. Silvestri, S. Reggiani, E. Gnani, A. Gnudi, and G. Baccarani, "Unified model for low-field electron mobility in bulk and SOI-MOSFETs with different substrate orientations and its application to quantum drift-diffusion simulations," in Proc. ULIS, 2008, pp. 129-132.

[19] Sentaurus Device Users' Manual, Synopsis Inc., Mountain View, CA, release a-2008.09 ed.

[20] A. Nathan and H. Baltes, Microtransducer CAD—Physical and Computational Aspects. New York: Springer-Verlag, 1999, ser. Computational Microelectronics.

[21] S. Dhar, H. Kosina, V. Palankovsky, E. Ungersboeck, and S. Selberherr, "Electron mobility model for strained-Si devices," IEEE Trans. Electron Devices, vol. 52, no. 4, pp. 527-533, Apr. 2005

[22] E. Gnani, S. Reggiani, M. Rudan, and G. Baccarani, "On the electrostatics of double-gate and cylindrical nanowire MOSFETs," J. Comput. Electron., vol. 4, no. 1/2, pp. 71-74, Apr. 2005.

[23] S. Takagi, A. Turiumi, M. Iwase, and H. Tango, "On the universality of inversion layer mobility in Si MOSFETs: Part II-Effects of surface orientation," IEEE Trans. Electron Devices, vol. 41, no. 12, pp. $2363-$ 2368, Dec. 1994.

[24] G. Baccarani, E. Gnani, A. Gnudi, S. Reggiani, and M. Rudan, "Theoretical foundations of the quantum drift-diffusion and density-gradient models," Solid State Electron., vol. 52, no. 4, pp. 526-532, Apr. 2008.

[25] L. Lucci, P. Palestri, D. Esseni, L. Bergagnini, and L. Selmi, "Multisubband Monte Carlo study of transport, quantization, and electron-gas degeneration in ultrathin SOI n-MOSFETs," IEEE Trans. Electron Devices, vol. 54, no. 5, pp. 1156-1164, May 2007.

[26] B. Goebel, D. Schumann, and E. Bertagnolli, "Vertical N-channel MOSFETs for extremely high density memories: The impact of interface orientation on device performance," IEEE Trans. Electron Devices, vol. 48, no. 5, pp. 897-906, May 2001.

[27] S. M. Sze, Physics of Semiconductor Devices. New York: WileyInterscience, 1981.
[28] F. Stern and E. Howard, "Properties of semiconductor surface inversion layers in the electric quantum limit," Phys. Rev., vol. 163, no. 3, pp. 816835, Nov. 1967.

[29] S. Takagi, J. Koga, and A. Toriumi, "Mobility enhancement of SOI MOSFETs due to subband modulation in ultrathin SOI films," Jpn. J. Appl. Phys., vol. 37, no. 3, pp. 1289-1294, Mar. 1998.

[30] K. Uchida, A. Kinoshita, and M. Saitoh, "Carrier transport in (110) nMOSFETs: Subband structure, non-parabolicity, mobility characteristics, and uniaxial stress engineering," in IEDM Tech. Dig., 2006, pp. 1-3.

[31] C. Jacoboni and L. Reggiani, "The Monte Carlo method for the solution of charge transport in semiconductors with applications to covalent materials," Rev. Mod. Phys., vol. 55, no. 3, pp. 645-705, Jul.-Sep. 1983.

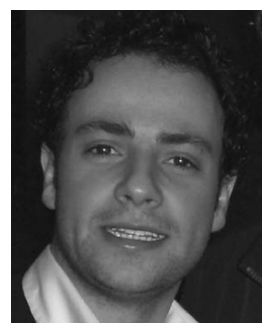

Luca Silvestri received the B.S. and M.S. de grees in electrical engineering from the University of Bologna, Bologna, Italy, in 2004 and 2007, respectively.

Since May 2007, he has been with the Advanced Research Center for Electronic Systems (ARCES), University of Bologna, and since January 2008, he has been in the European Doctorate program in Information Technology at ARCES, working in the development of physical models and simulation tools for nanoscale devices. Since October 2009, he has been a Visiting Researcher with the Microwave Laboratory, Université Catholique de Louvain, Louvain-la-Neuve, Belgium, working on modeling and simulation of Silicon Nanowires for sensor applications.

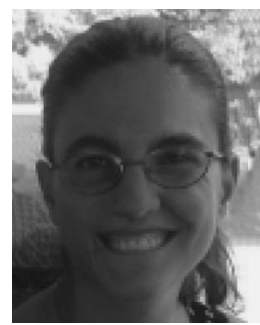

Susanna Reggiani (M'07) received the Laurea and $\mathrm{Ph} . \mathrm{D}$. degrees in electrical engineering from the University of Bologna, Bologna, Italy, in 1997 and 2001, respectively.

Since April 1997, she has been with the Department of Electronics (DEIS), University of Bologna working on the numerical simulation of semiconductor devices and the simulation of electron transport in nanoscale devices applied to quantum computing. In 2001, she became a Research Associate at the University of Bologna. She is currently with the Advanced Research Center for Electronic Systems.

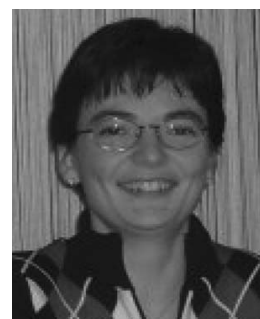

Elena Gnani (M'98) received the Laurea and Ph.D. degrees in electrical engineering from the University of Bologna, Bologna, Italy, in 1999 and 2003 respectively.

Since October 1999, she has been with the Department of Electronics (DEIS), University of Bologna, working on the physics of carrier transport and numerical analysis of semiconductor devices. She is currently with the Advanced Research Center for Electronic Systems.

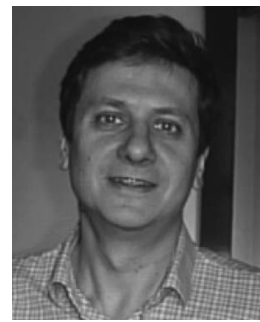

Antonio Gnudi (M'98) received the Laurea degree in electrical engineering and the Ph.D. degree in electrical and computer science from the University of Bologna, Bologna, Italy, in 1983 and 1989, respectively.

From 1989 to 1990 , he was a Visiting Scientist at IBM T.J. Watson Research Center, Yorktown Heights, NY, engaged in the development of advanced physical models and numerical simulation tools for charge transport in semiconductor devices. He became a Research Assistant in 1990 and an Associate Professor of electronics in 1998 at the University of Bologna, where he was involved in research and teaching activities concerning the design of analog CMOS circuits for RF applications and the modeling, design, and characterization of RF-MEMS devices for wireless applications. His current research interests include numerical simulation of nanometric devices, including semiclassical as well as full-quantum transport, and efficient algorithms for their solution. 


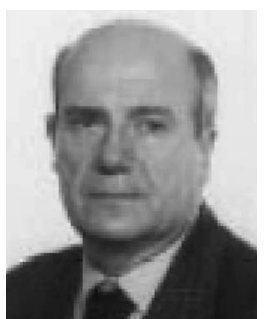

Giorgio Baccarani (S'68-M'80-SM'92-F'00) received the Dr.Ing. degree in electrical engineering and the Ph.D. degree in physics from the University of Bologna, Bologna, Italy, in 1967 and 1969, respectively.

In 1969, he joined the Bell Laboratories, Murray Hill, NJ, as a limited-term Member of the Technical Staff, working on electron-device processing. In 1970, he became a Research Assistant at the University of Bologna, where he investigated the physical properties of MOS structures and the transport effects in semiconductor materials and devices. Since 1972, he has been teaching an annual course on quantum electronics. In 1980, he became a Full Professor of digital electronics at the University of Bologna. He is one of the initial proposers and the Director of the Advanced Research Center for Electronic Systems, University of Bologna, founded in 2001. In 1981, he was on a one-year assignment at the IBM T.J. Watson Research Center, Yorktown Heights, NY, where he investigated the feasibility of a $1 / 4$ micrometer MOS process from the standpoint of the physical limitations affecting the device performance. In 1983 and 1989, he was again a Visiting Scientist at IBM, investigating advanced physical models for device simulation, and silicon-based heterojunction bipolar transistors, respectively. Since 1983, he has been heading a group involved in numerical analysis of semiconductor devices, acting as partner leader in the context of several EEC-supported Projects in the area of CAD for VLSI. His current research interests include numerical-device simulation, synthesis of analog circuits, analog and digital architectures for image processing, and integrated circuit design. 